\title{
An Animated Pedagogical Agent supporting student learning using performance analysis from a Fuzzy Agent
}

\section{Gustavo Mateus ${ }^{1}$, Beatriz Wilges ${ }^{2}$, Daniel Diniz ${ }^{4}$, Silvia Nassar ${ }^{1}$, Vilson Wronscki $^{1}$, Renato Cislaghi ${ }^{3}$}

${ }^{1}$ Programa de Pós-Graduação em Ciência da Computação - Universidade Federal de Santa Catarina (UFSC)

${ }^{2}$ Programa de Pós-Graduação em Engenharia e Gestão do Conhecimento - Universidade Federal de Santa Catarina (UFSC)

${ }^{3}$ Programa de Pós-Graduação em Métodos e Gestão em Avaliação - Universidade Federal de Santa Catarina (UFSC)

Caixa Postal 88.040-900 - Florianópolis - SC - Brasil

${ }^{4}$ Universidade Federal de Minas Gerais (UFMG)

Caixa Postal 31270-901 - Belo Horizonte - MG - Brasil

\{gustavo.mateus, silvia.nassar,vilson.wronscki, renato.cislaghi@ufsc.br\}

\{beaw@inf.ufsc.br\}, \{danieldiniz@ufmg.br\}

\begin{abstract}
This paper presents a framework made up of agents that promote monitoring of the learning process in a Virtual Learning Environment (VLE) On the other hand. From the results of student performance in the VLE it was possible to perform the mapping of performance on formal tests. Having this mapping it is possible to evaluate whether the decision-making from the Fuzzy Agent (FA) is consistent with the intervention that the interface agent performs. The interface agent is an Animated Pedagogical Agent (APA) which has a specific behavior according to student performance, it is intended to transmit the messages to the student so it can track and monitor the whole learning process in the Virtual Learning Environment.
\end{abstract}

\section{General Information}

Many studies present an interest in how to best guide students while using a Virtual Learning Environment (VLE). Van, Erik and Schepers's studies (2008) show evidence that the success of a VLE depended on the acceptance and usage of the e-learning system. In addition, they also highlighted the fact that education program managers should not worry exclusively about the basic system project, but should also deal explicitly with the individual differences between each VLE user.

It was also observed in Chou and Liu's work (2005) that web-based technology has a positive impact in learning. In their studies, they presented data regarding the efficacy of a VLE in relation to traditional, face-to-face teaching method. Four critical factors were also identified, according to the authors: performance increase, higher teaching efficiency, higher student satisfaction levels, and learning.

Similar studies aim to find constructs in student-oriented teaching on web-based systems. Ke and Kwak (2013) formulated hypothesis regarding student-oriented 
teaching on web-based systems and tested them using structural equation modeling. According to these authors, there are five main constructs in online courses: student interest, active learning, authentic learning, student autonomy, and the student's personal competency with computer technologies. These authors also postulate that these factors are statistically significant to the students' personal satisfaction with their learning process.

Another factor that can boost VLEs lies within the usage of animated agents. According to Behrend and Thompson (2012), animated agents have the potential to increase student interest, engagement, and learning during an online course. Agents therefore come to act as customized tutors.

The objective of this research is to find an adequate way to accompany and stimulate student learning in a VLE. Additionally, we have decided to analyze student performance results on tests applied on their daily classroom environment against their performance while using the VLE, verifying if the Animated Pedagogical Agent (APA) evaluation and intervention corresponded with student general performance on traditional, face-to-face tests.

This article is organized as follows: the next section introduces the framework proposed to keep track of student learning in a VLE. The following sections bring details on agents involved in this framework: the Fuzzy Agent (FA) and the APA. In the fifth section, the tool used as a means of acquiring information, through user input, regarding the structure of traditional tests applied at the classroom is introduced. In the sixth section some analyses made using performance determined by FA in the VLE and data from performance by a group of students in traditional tests are presented. Finally, conclusion and final remarks of this research shall be presented.

\section{A framework to monitor student learning progress}

This research presents a study regarding performance data collected from a group of students. Performance was considered and compared between two sources: while inside the VLE, and also from traditional, face-to-face tests applied in classrooms. An interface agent was used as a means of best directing each student's individual learning process through specific messages, alerts, and even an eventual praise, when duly deserved.

Thus, this research employed two different kinds of agents: A Fuzzy Agent (FA) and an Animated Pedagogical Agent (APA). The FA is a cognitive agent which makes use of data acquired from the VLE's theoretical and practical tests to determine how the APA should behave, making the APA a reactive agent in this process. Figure 1 illustrates the framework proposed for monitoring student progress. 


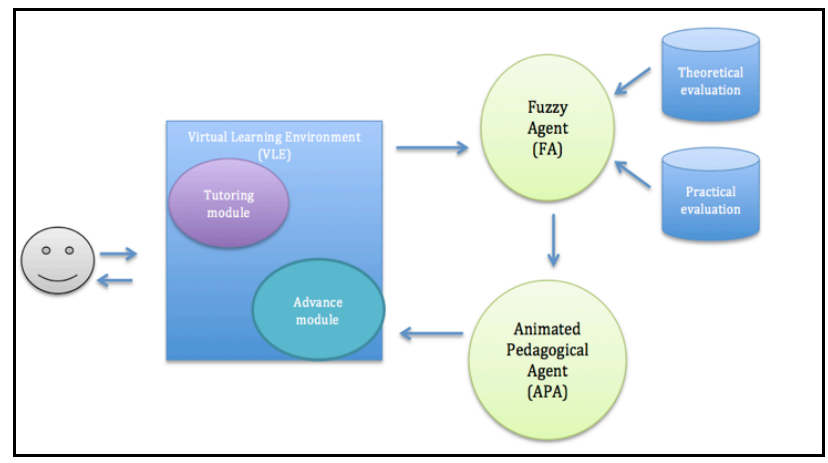

Figure 1. Framework for student progress follow-up

We choose the Virtual Learning Environment (VLE) which is used for semipresent teaching in the Statistics class for Engineering students in an university. This VLE is based on significant learning, and by using it the student has the possibility of working research data in two different learning modules: tutoring and advanced.

Initially, students work with the tutoring module, where statistic content is presented while guided by a Concept Map (CM). Each step the student takes is linked to a datasheet, which can be related to their research or to an example made available by a teacher in the VLE.

In this tutoring module, students have complete freedom to choose their learning paths. The CM shows and enables to the student access to the complete path that one given interaction follows until it reaches its final statistical result, also showing other paths which the system can follow, in case different answers are given on each step of the process. This feature allows easy understanding by students to follow each step of the process, also presenting easy behavior, progress, and learning curve tracking and monitoring.

On the other hand, the VLE advanced module allows students to apply their desired statistical analysis directly on their data, without support by a tutoring module; in other words, without the overwhelming presence of the CM. Usually, this module is only used by students which have learned how to choose and apply their desired analysis in the tutoring module, and after feeling confident enough to work with it.

\section{Fuzzy Agent (FA) implementation}

Studies by Mateus et al. (2011) already pointed out the need to find an adaptive module for individual students in a VLE according to their own performance, and a diffuse modeling for the VLE in relation to the data obtained regarding class performance results have already been detailed in studies by Wilges et al. (2012). Besides, other papers by Mateus et al. (2010) had already tried to recognize student profile through Multi-Agent System (MAS) and fuzzy logic.

In this study, student performance within the VLE is related to traditional classroom tests. VLE student development is determined by two input variables (practicalPerformance and theoreticalPerformance), and an output variable (generalEvaluation). All these variables are sent to a Fuzzy Agent (FA) where they are associated with a set of pertinence functions. These functions are composed by three linguistic performance variables: insufficient, regular, and good. 


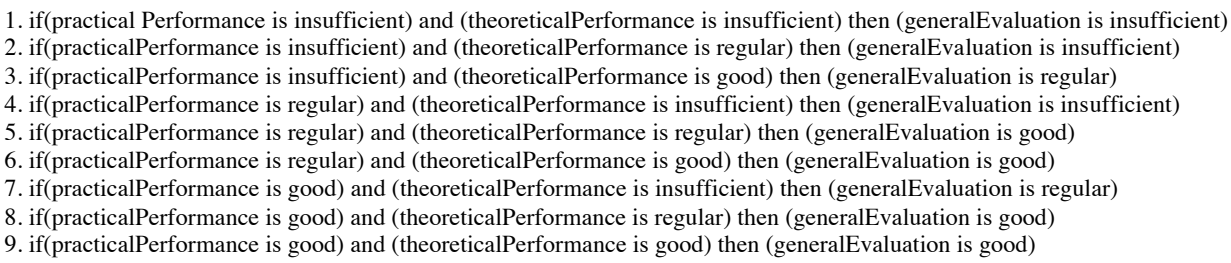

Figure 2. Rules database

The inference engine controller used was that of Mamdani and Assilian (1999), implemented with "minimal" implication operator and the adopted defuzzification method was the center of gravity method [Li and Gupta 1995]. Nine inference rules were defined to cover all linguistic variables used, and each rule is composed by the "and" operator associated with the "minimal" method. Aggregation between rules is made by the "maximal" method. Figure 2 presents the rules database.

In order to activate the inference engine, a file with the group of entries, which contains student theoretical performance and practical performance entries, is used. This file is then submitted together with the fuzzy model (FIS file) to the Fuzzy Agent (FA), and the defuzzyfied output variable (generalEvaluation) is generated.

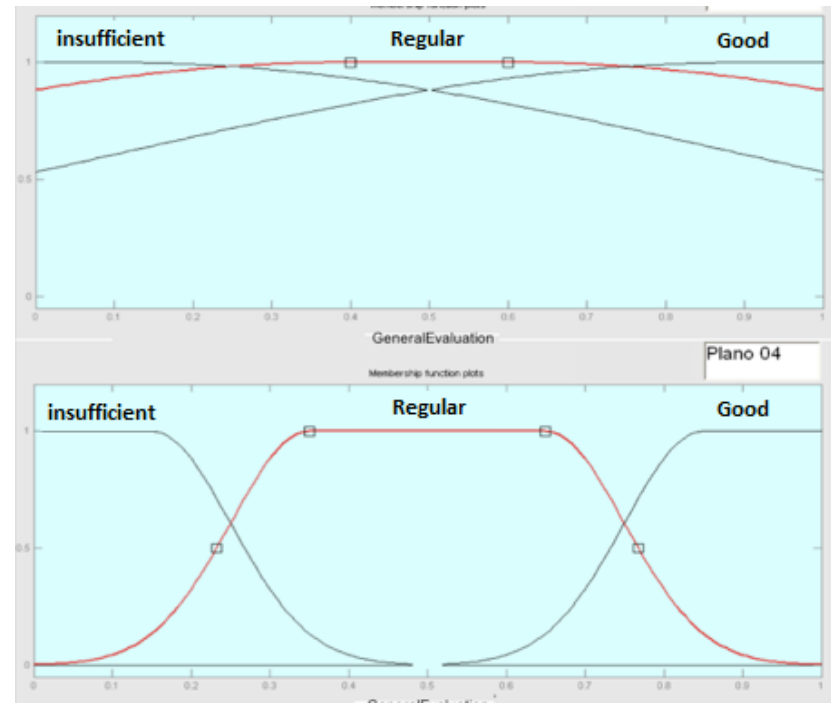

Figure 3. Fuzzy library levels

The Fuzzy Agent (FA) makes use of a group of four pertinence function levels, which belongs to a fuzzy library. Each level was defined with different values for pertinence function parameters. This way, it was possible to develop a library, which allowed for the application of a group of pertinence functions which varies dynamically according to the student's adaptation and performance. In other words, we have implemented pertinence function levels which alternate between a higher level of uncertainty and a lower level of uncertainty regarding the student's learning level. The objective of each level is to categorize the student inside the insufficient, regular, and good category groups. Figure 3 shows the first and last levels of the fuzzy library.

Students change level in this fuzzy library as soon as grades reach a value $60 \%$ above the minimum value possible in their current level, which makes the system 
update their fuzzy level in the Database (DB) to the next level. This minimal value for general evaluation is an institutional parameter and was adopted as $60 \%$, as in this institution the minimal grade for approval is 6.0.

\section{Animated Pedagogical Agent (APA) implementation}

In this research, the interface agent is represented by an animated pedagogical agent (APA), which sends verbal messages to the students involved with the VLE. The purpose of this agent is to conduct the students so they can make the best use of the resources available, displaying customized messages according to evaluated performance while using the VLE.

According to Baylor and Kim (2005), intelligent agents are used in a wide variety of organizational environments, be it educational or commercial. Animated pedagogical agents (APAs) are a specific class of agents which are represented by an animal or human figure inside the VLE, and conceived to make learning easier. Figure 4 presents a teacher character, chosen to be the APA of this VLE. This character was chosen through a poll among all students who have used the VLE at least once.

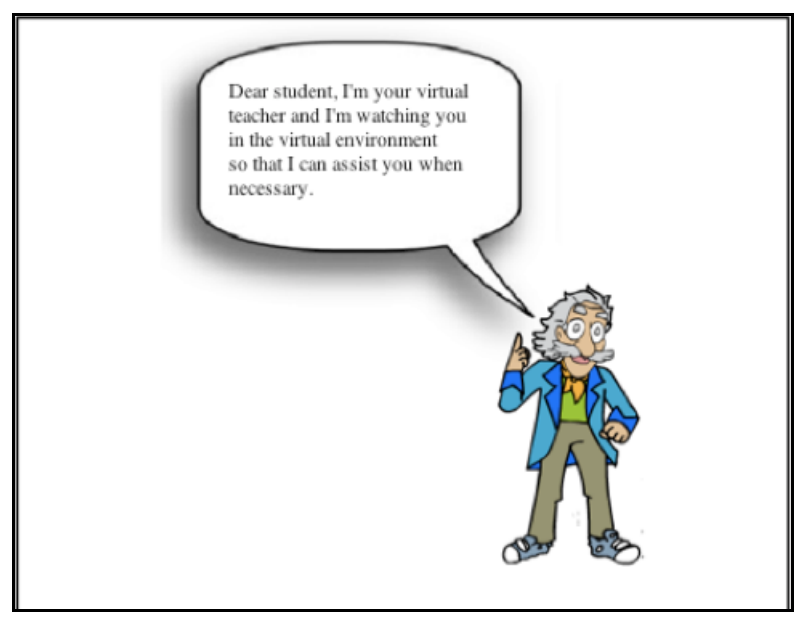

Figure 4. The VLE Animated Pedagogical Agent

This character acts in different situations, according with student learning profile. According to Rabello, Reategui and Costi (2007), interface agents usually act as support for a specific application, making it easier for the user to work with it. The character present in VLE also has this same purpose, advising, guiding the teaching and learning process, and being a facilitator inside the VLE, in general. The APA offers suggestions and orientations according to student performance observed on the environment. Its main role consists of making users feel like they are being accompanied, and is therefore responsible for both praising students in situations where they perform according to what is expected, as well as warning them of incorrect answers or thinking processes. Figure 5 presents two examples of described interventions made by the character while accompanying the student. 


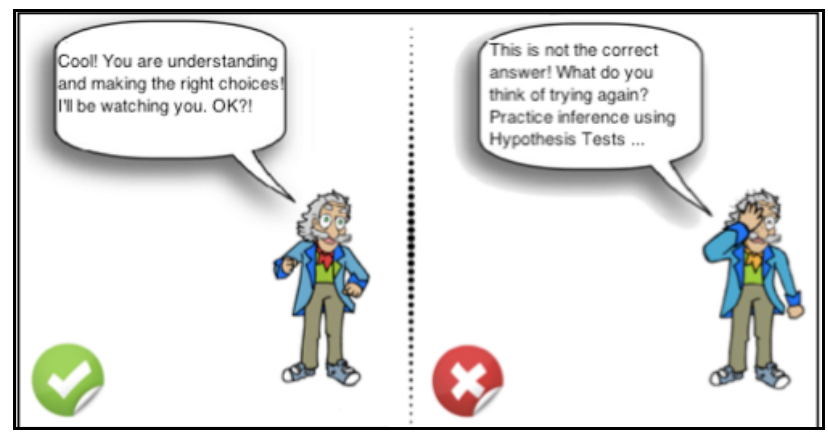

Figure 5. Situations that may come up while

The animated pedagogical agent is also responsible for redirecting students to a module which is more adequate to their performance, where they can continue to learn and work. This procedure occurs in cases where students reach the fourth level of the fuzzy library. In these cases, the FA supposes that the student is ready to work directly with a more advanced module; in other words, without the APA tutoring during the analyses made by the student. In this more advanced module of the VLE, students can focus on statistical analyses and also benefit from an environment without functionalities oriented to students without higher previous knowledge of the content. Figure 6 illustrates how the character behaves in a situation where students have achieved this last level of the fuzzy library.

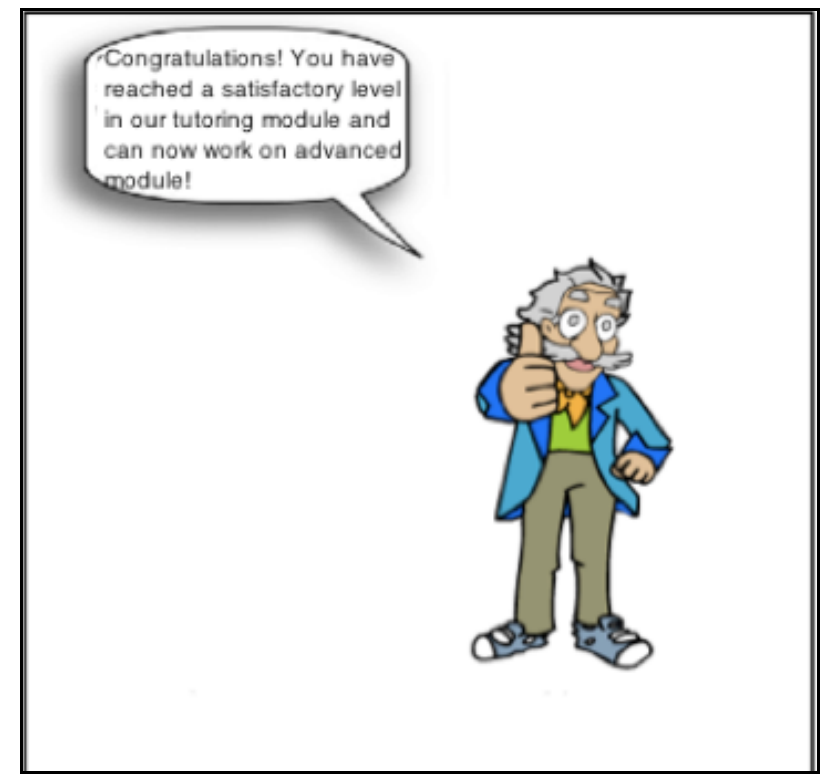

Figure 6. APA redirecting the student to the advanced

\section{Evaluating learning results through tests}

In order to confirm if the FA was capable of correctly determining student learning levels, data extracted from student performance at tests applied in classrooms has been used. In order to facilitate correlating virtual and physical data, a VLE tool in which classroom tests could be digitized to the system has been implemented. This way, massive amounts of data were available to analyze and correlate with each student's personal result obtained through the effective use of the VLE. 
As data resulting from the effective use of the VLE was already stored and made available at the same time students use and practice with the various online exercises, this tool was necessary to enable input both details regarding the various questions present in the conventional classroom tests as well as corresponding grades obtained by each individual student at each individual question. Therefore, when digitalizing a test, each question must be classified by theme and difficulty level in order to store data with thinner granularity and therefore enriching future analyses. Figure 7 shows the insertion of a question according to its theme, concept, and difficulty level.

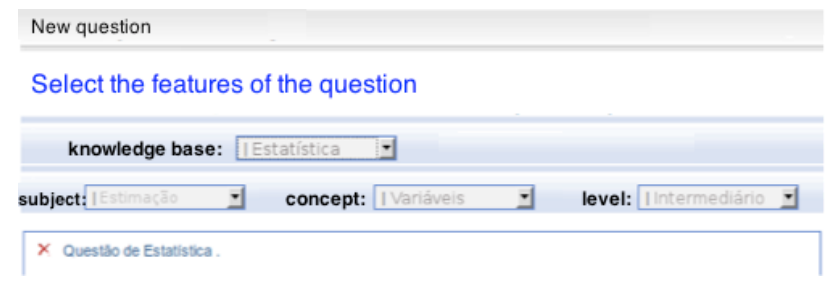

Figure 7. Question properties

Teachers should categorize each question of a test according to theme, concept, and difficulty level. Student answers during experimentation with the VLE are promptly stored within the database. With this, it is possible to make a parallel that evaluates if FA beliefs and APA interactions really contribute to student learning process and if they do, how this can reflect on classroom grades.

\section{Performance results obtained from the VLE and from tests}

Three formal, face-to-face evaluations were applied during the semester. For the sake of this study we have observed the structure and results from the second test applied in semester 2012/2 with a group of Engineering students. Figure 8 shows the difficulty levels of each of the questions in this test.

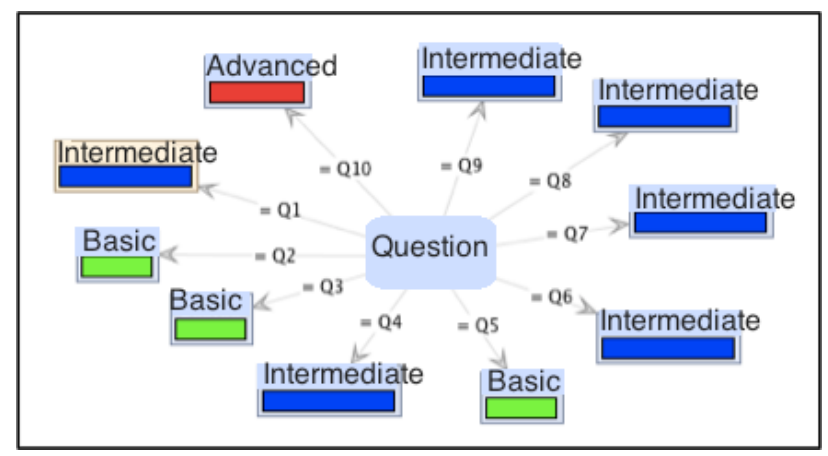

Figure 8. Question properties

We calculated average grades scored by students in each question, by difficulty level. The average of correct answers for the basic level was $81 \%, 82 \%$ for the intermediate level, and $63.8 \%$ for questions ranked as advanced. Figure 9 shows configuration of average values scored by students at each question, according to their difficulty level as shown in Figure 8. It can be noticed that questions Q1, Q4 and Q6, previously registered as questions of intermediate level, have superior averages than basic questions. Notice that the grade values of these questions are an average between the values of 0 and 1, as Figure 9 shows. 


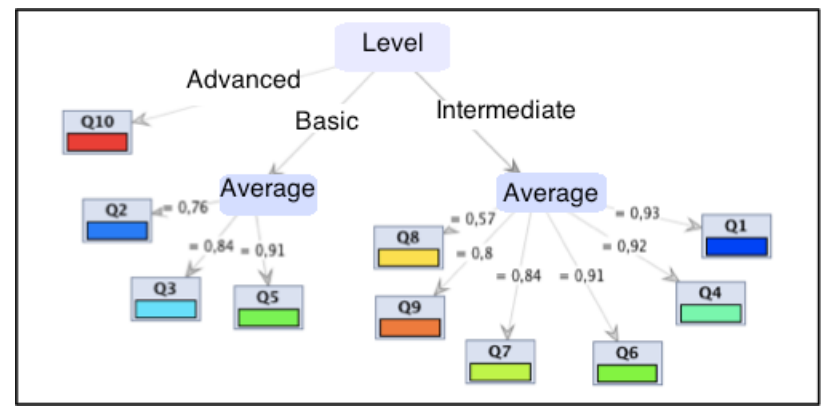

Figure 9. Average performance in each question

Figures 10 show student performance at the FA levels, accompanied by values scored during formal face-to-face testing, according to the questions and difficulty levels presented. The main objective of this figure is to correlate student movement between fuzzy levels library to student results from the second formal test.

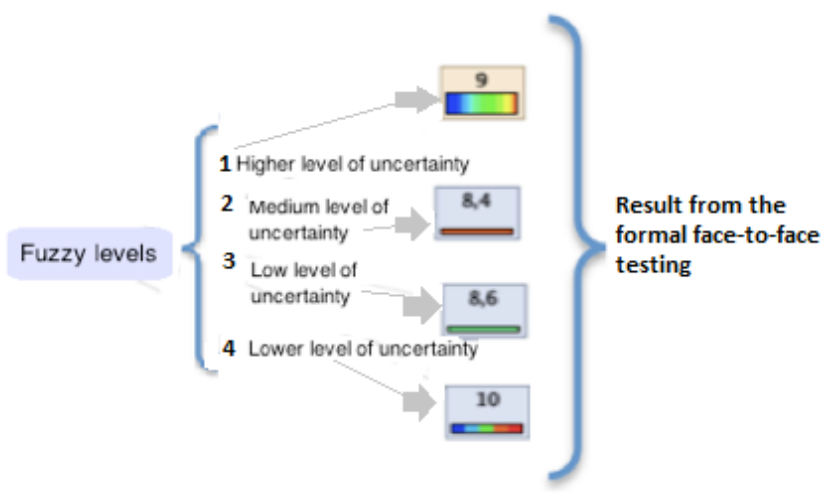

\section{Figure 10. Correlation between learning noticed by FA and student grade values from formal testing.}

The results in Figure 10 show that students in the last level of the library, with the lowest level of uncertainty, tend to have higher scores on the formal test. This is because these students achieve a higher number of correct questions in practicalPerformance and theoreticalPerformance from the VLE. Thus, these students are better prepared and present a better result on the formal test.

\section{Conclusions}

Results obtained from APA inference using FA logic during performance analysis processes configure a richer and more rewarding learning experience for students. This can be clearly seen reflected on the results coming from formal, face-to-face tests, making it possible to evaluate if actions implemented in the VLE by FA are in accordance with student learning requirements.

This way, it is possible to analyze whether the framework proposed as a means of measuring performance while using a VLE is fulfilling its role and making the entire environment easier for each student. In addition, the application of a diffuse approach in 
different scenarios and moments of the learning process enables a more flexible analysis, making it possible to have an APA with customized messages according to each student. In other words, the learning process is not treated in a deterministic manner, such as "passed" or "failed", but with diffuse values that can vary from 0 to 1 . After each stage in a fuzzy library level is completed, the APA has enough data for analyses, therefore becoming capable of making assumptions regarding student learning accomplishments. The entire process involving collecting information regarding student performance occurs in a very transparent manner, in each of the student's actions while inside the VLE.

\section{References}

Baylor, A. L.; Kim, Y. Simulating instructional roles through pedagogical agents. International Journal of Artificial Intelligence in Education. Vol. 15, no. 2, pp. 95115, Apr. 2005. Available: http://dl.acm.org/citation.cfm?id=1434925.1434927

Behrend, Tara S.; Thompson, Lori Foster. Using animated agents in learner controlled training: the effects of design control," International Journal of Training and Development, vol. 16, no. 4, pp. 263-283, 2012.

Chou, Shih-Wei; Liu, Chien-Hung. Learning effectiveness in a web-based virtual learning environment: a learner control perspective. Journal of Computer Assisted Learning, vol. 21, no. 1, pp. 65-76. 2005.

Ke, Fengfeng and Kwak, Dean. Constructs of student-centered online learning on learning satisfaction of a diverse online student body: A structural equation modeling approach. Journal of Educational Computing Research, 2013.

Li, Hua; Gupta, Madan M. Fuzzy Logic and Intelligent Systems. Springer, vol. 3. 1995.

Mateus, G. P.; Wilges, b.; Nassar, S. M., R. A. Silveira; Bastos, R. Reconhecendo as características dos estudantes por meio de um SMA e lógica fuzzy. RENOTE, vol. 8, no. 2, 2010.

Mateus, G. P.; Wilges b.; Nassar S. M.; Wronscki, V., Bastos, R. C. Um módulo adaptativo para AVA baseado no desempenho do estudante. In Anais do Simpósio Brasileiro de Informática na Educação, vol. 1, no. 1, 2011.

Mamdani, E. H.; Assilian, S. An experiment in linguistic synthesis with a fuzzy logic controller. International journal of man-machine studies. Vol. 51, no. 2, pp. 135-147, Aug. 1999. Available: http://dx.doi.org/10.1006/ijhc.1973.0303

Rabello, R. dos Santos; Reategui E.; Costi, J. C. G. Um agente de interface aplicado ao ensino de equações de primeiro grau. RENOTE, vol. 5, no. 2, 2007.

Van Raaij, Erik M; Schepers, Jeroen JL. The acceptance and use of a virtual learning environment in china. Computers \& Education, vol. 50, no. 3, pp. 838-852, 2008.

Wilges, B.; Mateus, g.; Nassar, s.; Bastos, R. Fuzzy modeling built through a data mining process," Latin America Transactions, IEEE (Revista IEEE America Latina), vol. 10, no. 2, pp. 1622-1626, 2012. 